\title{
Synthesis of L-Ascorbic Acid Lactone Derivatives
}

\author{
Li-Dong Shao $\cdot$ Ya-Nan Wu $\cdot$ Jun Xu $\cdot$ Juan He $\cdot$ Yu Zhao $\cdot$ Li-Yan Peng $\cdot$ \\ Yan Li $\cdot$ Yu-Rong Yang $\cdot$ Cheng-Feng Xia $\cdot$ Qin-Shi Zhao
}

Received: 13 April 2014/ Accepted: 28 April 2014/Published online: 21 May 2014

(C) The Author(s) 2014. This article is published with open access at Springerlink.com

\begin{abstract}
A small focused library which comprised of L-AA lactone derivatives was built with a facile method. This reported method was optimized by modifying the acidity of the solvent. As a result, 12 L-AA lactones were synthesized. Among these lactones, lactones 8-12 were new compounds. The cytotoxicity of these synthetic compounds were investigated.
\end{abstract}

Keywords L-Ascorbic acid lactone $\cdot$ Cytotoxicity $\cdot$ Focused library

\section{Introduction}

L-Ascorbic acid (L-AA), one form of vitamin C, plays an important role in both plant and animal physiology. The foremost biologically functions of L-AA are centred around the antioxidant properties. Considerable evidence has been accruing in the last two decades about the importance of L-AA not only in protecting the plant from oxidative stress, but also in protecting mammals from various chronic diseases that have their origins in oxidative stress [1]. Derivatives of LAA were found showing wide range of bioactivities including antiviral [2-5], cytotoxicity [6], inhibitory activities against tyrosinase-catalyzed melanin formation [7], increasing skin

L.-D. Shao · Y.-N. Wu · J. Xu · J. He · Y. Zhao $\cdot$ L.-Y. Peng · Y. Li · Y.-R. Yang · C.-F. Xia (ه) · Q.-S. Zhao (ه)

State Key Laboratory of Phytochemistry and Plant Resources in

West China, Kunming Institute of Botany, Chinese Academy of

Sciences, Kunming 650201, China

e-mail: xiachengfeng@mail.kib.ac.cn

Q.-S. Zhao

e-mail: qinshizhao@mail.kib.ac.cn

L.-D. Shao · Y.-N. Wu · J. Xu

Graduate School of the Chinese Academy of Sciences,

University of Chinese Academy of Sciences, Beijing 100049,

China permeability [8, 9], and neurotropic activity [10]. Among them, octanoyl-6- $O$-ascorbic acid could enhance the solubility of many poorly water soluble drugs [11]. Because of these properties, L-AA derivatives were applicable in cosmetics and medicine [12, 13].

Many bioactive L-AA derivatives were found in nature [14-16]. For example, bioactive-oriented isolation of dilaspirolactone aglycon (1) and delesserrine (2) (Fig. 1) from Delesseriaceae family were reported [17, 18]. Our research group are interested in fern plants for a long time. A lot of species were systematically studied towards chemical components and their bioactivities [19-23], which led to the isolate of dichotomains A and B (3, 4) (Fig. 1), two LAA derivatives, from Dicranopteris dichotoma. And dichotomain B (4) was confirmed as a weak HIV-1 inhibitor [24]. These compounds with a fragment of L-AA lactone showed different bioactivities. Attracted by this difference and the unique structure of L-AA derivatives, we would like to build a small focused library of L-AA lactone derivatives to explore their bioactivities.

\section{Results and Discussion}

Tang et al. [25] reported a short total synthesis of L-AA lactone compounds leucodrin and leudrin through a 
<smiles>O=C1C[C@@](O)(c2ccc(O)cc2)[C@@]2(OCC(O)[C@H]2O)C1O</smiles>

1, Dilaspirolactone aglycon<smiles>CO[C@]1(Cc2ccc(O)cc2)C(=O)OC2C(O)COC21</smiles>

2, delesserrine

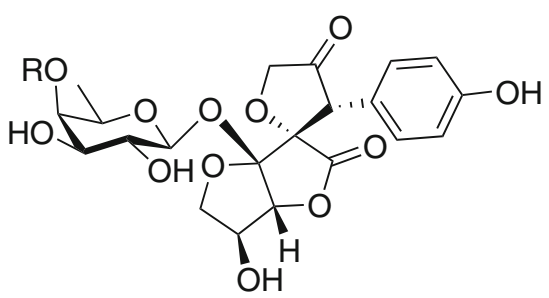

3, $\mathrm{R}=\mathrm{Ac}$, dichotomain $\mathrm{A}$ 4, $\mathrm{R}=\mathrm{H}$, dichotomain $\mathrm{B}$

Fig. 1 Natural L-AA lactone derivatives<smiles>[R9]OC1=C(O)[C@@H]([C@H](O)CO)OC1=O</smiles><smiles>[R6]C(O)c1ccc(O)c(Br)c1</smiles><smiles>[C-]#CO</smiles>

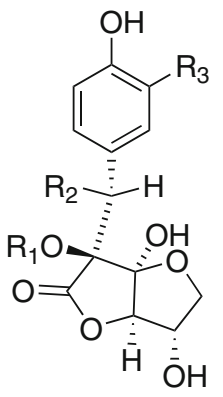<smiles>CCC</smiles>

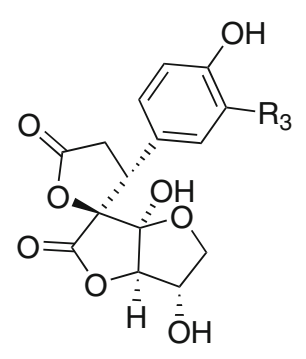

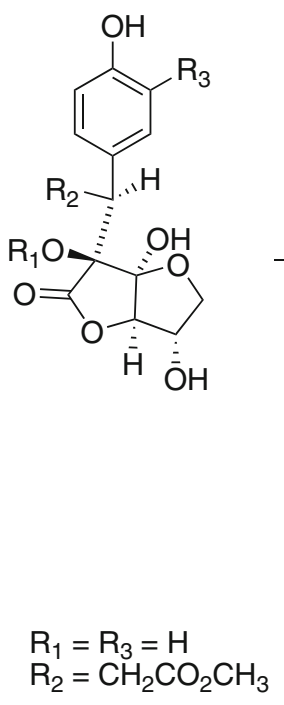

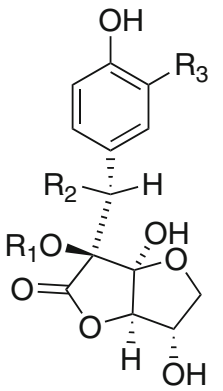

Scheme 1 A synthetic route to L-AA lactone derivatives reported by Poss et al organocatalystic 1,4 -conjugate addition of L-AA to $\alpha, \beta$ unsaturated aldehydes. Although it effectively synthesized $5 / 5 / 5$ spirodilactone L-AA derivatives, it could not access to other L-AA lactone derivatives. Poss et al. [26] reported that treating L-AA with different 4-hydoxy benzyl alcohols in hot water resulted in L-AA lactone derivatives (scheme 1). With this method, we obtained some L-AA lactone derivatives $(\mathbf{5}, \mathbf{7}, \mathbf{1 3}, \mathbf{1 4}, \mathbf{1 5}$, Fig. 2), but a number of L-AA lactone derivatives $(\mathbf{6}, \mathbf{8}, \mathbf{9}, \mathbf{1 0}, \mathbf{1 1}, \mathbf{1 2}, \mathbf{1 6}$, Fig. 2) could not formed by using this methods. The failure probably was caused by acidity of the solvent [25, 27], so, we modified the condition by applying the phosphate-citrate buffer solution (PH 5.0) as solvent. As a result, compounds $\mathbf{6}, \mathbf{8}, \mathbf{9}, \mathbf{1 0}, \mathbf{1 1}, \mathbf{1 2}, \mathbf{1 6}$ were successfully synthesized.

All 4-hydroxy benzyl alcohols were synthesized by reduction of corresponding aldehydes with $\mathrm{NaBH}_{4}$ except B9 and B10 (Table 1). Without following the Ref. [26], 4-hydroxy benzaldehyde was protected with Bn group, and then reacted with methyl acetate through an aldol condensation. At last, removal of Bn group gave B9 in $89 \%$ yield. It is interesting that $\mathbf{B 1 3}$ could not react with L-AA to yield lactone compound. However, it worked with methyl ether in stead of ethyl ether. An air oxidative product B14 was detected in methanolysis reaction of $\mathbf{B 1 3}$, which was reducted by $\mathrm{NaBH}_{4}$ to afford $\mathbf{B 1 0}$ (Scheme 2).

With all designed 4-hydoxy benzyl alcohols in hand, we built a small focused library which contained L-AA lactone drivatives 5-16. We found that 4-hydoxy benzyl alcohols like B1, B4, B5, B7, and B11 with good water solubility could react well with L-AA to give lactone derivatives in good yield except B3. This might be that two phenolic hydroxyl groups in $\mathbf{B 3}$ made it be easily oxidized by air. B12 could not react with L-AA in all conditions applied in this article, probably because the reactivity of lone pair electron at $\mathrm{S}$ atom of $\mathbf{B 1 2}$ was lower than that of phenol hydroxyl. So, it could not react like other 4-hydoxy benzyl alcohols. Furthermore, all L-AA lactones were obtained as a single compound except lactones $\mathbf{9}$ and $\mathbf{1 0}$ which were mixtures of two isomers at benzylic position. ${ }^{1} \mathrm{H}$ NMR indicated their ratio is about 10:1 for $\mathbf{9}$ and 3.4:1 for 10, and we found that one isomer of $\mathbf{9}$ and $\mathbf{1 0}$ were unstable 
<smiles>O=C1O[C@H]2[C@@H](O)CO[C@]2(O)[C@@]1(O)Cc1ccc(O)cc1</smiles>

5<smiles>CC(c1ccc(O)cc1)[C@]1(O)C(=O)O[C@H]2C(O)CO[C@]21O</smiles>

9<smiles>O=C1C[C@]2(OC1=O)O[C@@H]1[C@@H](O)CO[C@@H]1[C@@H]2O</smiles>

13<smiles>COc1ccc(C[C@@]2(O)C(=O)O[C@@H]3[C@@H](O)CO[C@H]32)cc1</smiles>

6<smiles>COc1ccc(C(C)[C@]2(O)C(=O)O[C@H]3[C@@H](O)CO[C@]32O)cc1</smiles>

10<smiles>COc1ccc([C@@H]2CC(=O)O[C@]23C(=O)O[C@H]2[C@@H](O)CO[C@]23O)cc1</smiles><smiles>O=C1O[C@H]2[C@@H](O)CO[C@]2(O)[C@]1(O)Cc1ccc(O)c(O)c1</smiles>

7<smiles>COc1cc(C[C@@]2(O)C(=O)O[C@@H]3[C@@H](O)CO[C@@H]32)ccc1O</smiles>

11<smiles>COC(=O)C[C@@H](c1ccc(O)cc1)[C@@]1(O)C(=O)O[C@@H]2[C@@H](O)CO[C@]21O</smiles><smiles>O=C1O[C@H]2[C@@H](O)CO[C@]2(O)[C@@]1(O)Cc1ccc2c(c1)OCO2</smiles>

8<smiles>COc1cc(C[C@]2(O)C(=O)O[C@@H]3[C@@H](O)CO[C@]32O)cc(Br)c1O</smiles>

12<smiles></smiles>

Fig. 2 Synthetic L-AA lactone derivatives

and could transform from semi-ketal into ketone at C3 position at room temperature or under NMR condition spontaneously.

All synthetic L-AA lactone derivatives were evaluated on five human tumor cell lines, including HL-60, SMMC7721, A-549, MCF-7 and SW480, using 3-(4,5-dimethylthiazol-2-yl)-2,5-diphenyltetrazolium bromide (MTT) method (Table 2). Anticancer drug cisplatin (DDP) was used as the positive control. To our disappointment, none of these compounds showed cytotoxicity.

\section{Experiment Section}

\subsection{General Experimental Procedures}

HRESIMS were performed on a Agilent 6540 Q-TOF. ${ }^{1} \mathrm{H}$ and ${ }^{13} \mathrm{C}$ NMR spectra were recorded on Bruker Avance III400 and Bruker Avance III-600 MHz spectrometers. Chemical shifts $(\delta)$ were expressed in $p p m$ with reference to the TMS resonance. Column chromatography was performed using Silica gel [(200-300) mesh, Qingdao Marine Chemical, Inc, Qingdao, China]. Reactions were monitored by TLC and spots were visualized by heating the silica gel plates sprayed with $10 \% \mathrm{H}_{2} \mathrm{SO}_{4}$ in $\mathrm{EtOH}$.

\subsection{Synthesis of 4-Hydroxy Benzyl Alcohols}

\section{(B1-B8, B11-B12)}

4-Hydroxy benzyl alcohols B1-B8, B11-B12 were synthesized by reduction of corresponding commercial available aldehydes with $\mathrm{NaBH}_{4}$ in $\mathrm{MeOH}$ at $0{ }^{\circ} \mathrm{C}$ for $1-4 \mathrm{~h}$.

B3: Brown foam, $90 \%$ yields, ${ }^{1} \mathrm{H}$ NMR $(400 \mathrm{MHz}$, $\left.\mathrm{D}_{2} \mathrm{O}\right) \delta 6.50-6.56(\mathrm{~m}, 3 \mathrm{H}), 4.34(\mathrm{~s}, 2 \mathrm{H})$.

B5: Light yellow oil, $96 \%$ yields, ${ }^{1} \mathrm{H}$ NMR $(400 \mathrm{MHz}$, acetone-D6) $\delta 8.29(\mathrm{~s}, 1 \mathrm{H}), 7.20(\mathrm{~d}, J=8.4 \mathrm{~Hz}, 1 \mathrm{H}), 6.77$ $(\mathrm{d}, J=8.4 \mathrm{~Hz}, 1 \mathrm{H}), 4.75(\mathrm{~m}, 1 \mathrm{H}), 4.04(\mathrm{~d}, J=4 \mathrm{~Hz}, 1 \mathrm{H})$, $1.36(\mathrm{~d}, J=6.4 \mathrm{~Hz}, 3 \mathrm{H})$.

B7: White solid, $92 \%$ yields, ${ }^{1} \mathrm{H}$ NMR $(400 \mathrm{MHz}$, $\left.\mathrm{CDCl}_{3}\right) \delta 9.72(\mathrm{~s}, 1 \mathrm{H}), 7.57(\mathrm{~d}, J=1.6 \mathrm{~Hz}, 1 \mathrm{H}), 7.30(\mathrm{~d}$, $J=1.6 \mathrm{~Hz}, 1 \mathrm{H}), 6.48(\mathrm{~s}, 1 \mathrm{H}), 3.91(\mathrm{~s}, 3 \mathrm{H})$.

B8: White solid, $82 \%$ yields, ${ }^{1} \mathrm{H}$ NMR $(400 \mathrm{MHz}$, $\left.\mathrm{CDCl}_{3}\right) \delta 7.02(\mathrm{~d}, J=1.0 \mathrm{~Hz}, 1 \mathrm{H}), 6.79(\mathrm{~d}, J=1.0 \mathrm{~Hz}$, $1 \mathrm{H}), 5.86$ (br. s, 1H), 4.53 (s, 2H), 3.85 (s, 3H).

\subsection{Synthesis of 4-Hydroxy Benzyl Alcohols (B9, B10)}

B9: To a solution of 4-hydroxy benzldehyde (12.2 g, $0.1 \mathrm{~mol})$ in $100 \mathrm{~mL}$ acetone, $\mathrm{K}_{2} \mathrm{CO}_{3}(20.7 \mathrm{~g}, 0.15 \mathrm{~mol})$ and $\mathrm{BnCl}(12.6 \mathrm{~mL}, 0.11 \mathrm{~mol})$ was added, which was 
Table 1 Synthesis of L-AA lactone derivatives

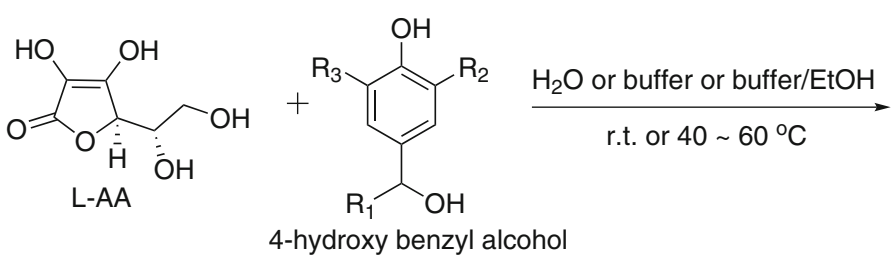<smiles>[R]c1cc(C([R])[C@]2(O)C(=O)O[C@@H]3[C@@H](O)CO[C@H]32)cc([R])c1O</smiles>

lactone tpye I<smiles>[R6]c1cc([C@@]23CC(=O)O[C@]2(O)[C@@H]2OC[C@H](O)[C@H]2OC3=O)cc([R])c1O</smiles>

lactone tpye II<smiles>O=C1O[C@H]2[C@@H](O)CO[C@]2(O)[C@]1(O)Cc1c[nH]c2ccccc12</smiles>

lactone tpye III \begin{tabular}{ll}
\hline Entry & 4-hydroxy benzyl alcohol \\
\hline 1 &
\end{tabular}

B1

2<smiles>COc1ccc(CO)cc1</smiles>

3<smiles>OCc1ccc(O)c(O)c1</smiles>

4<smiles>O=C(c1ccccc1)c1ccc2c(c1)OCO2</smiles>

5<smiles>CC(O)c1ccc(O)cc1</smiles>

B5

6<smiles>COc1ccc(C(C)O)cc1</smiles>

7

8<smiles>COc1ccc(CO)cc1Br</smiles><smiles>COc1cc(CO)cc(Br)c1O</smiles><smiles>CC(=O)CC(O)c1ccc(O)cc1</smiles>

B9

10

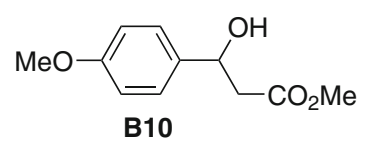

Condition

$\mathrm{H}_{2} \mathrm{O}, 50{ }^{\circ} \mathrm{C}, 50 \mathrm{~h}$

buffer, $40{ }^{\circ} \mathrm{C}, 72 \mathrm{~h}$

$6(53 \%)$

$\mathrm{H}_{2} 0,50{ }^{\circ} \mathrm{C}, 72 \mathrm{~h}$

$7(35 \%)$

buffer, $60^{\circ} \mathrm{C}, 36 \mathrm{~h}$

$8(72 \%)$

buffer, $60{ }^{\circ} \mathrm{C}, 60 \mathrm{~h}$

buffer, $60{ }^{\circ} \mathrm{C}, 72 \mathrm{~h}$

buffer, $40{ }^{\circ} \mathrm{C}, 48 \mathrm{~h}$

buffer, $60{ }^{\circ} \mathrm{C}, 72 \mathrm{~h}$

$\mathrm{H}_{2} 0,50{ }^{\circ} \mathrm{C}, 72 \mathrm{~h}$

$\mathrm{H}_{2} 0,50{ }^{\circ} \mathrm{C}, 72 \mathrm{~h}$

$14(30 \%)$
$10(42 \%) 3.4: 1$ of two benzylic siomers

$11(78 \%)$

$12(58 \%)$

$\mathbf{1 3}(35 \%) \mathbf{1 5}(40 \%)$ 
Table 1 continued

\begin{tabular}{lll}
\hline Entry & Condition & Product (yields) \\
\hline 11 & buffer/EtOH, r.t, $8 \mathrm{~h}$ & $\mathbf{1 6}(57 \%)$ \\
12 & $\begin{array}{l}\mathrm{H}_{2} \mathrm{O}, 50{ }^{\circ} \mathrm{C}, 72 \mathrm{~h} \\
\text { buffer, } 60{ }^{\circ} \mathrm{C}, 72 \mathrm{~h} \\
\text { buffer/EtOH, r.t., } 72 \mathrm{~h}\end{array}$ & $\mathrm{nr}$ \\
\hline
\end{tabular}

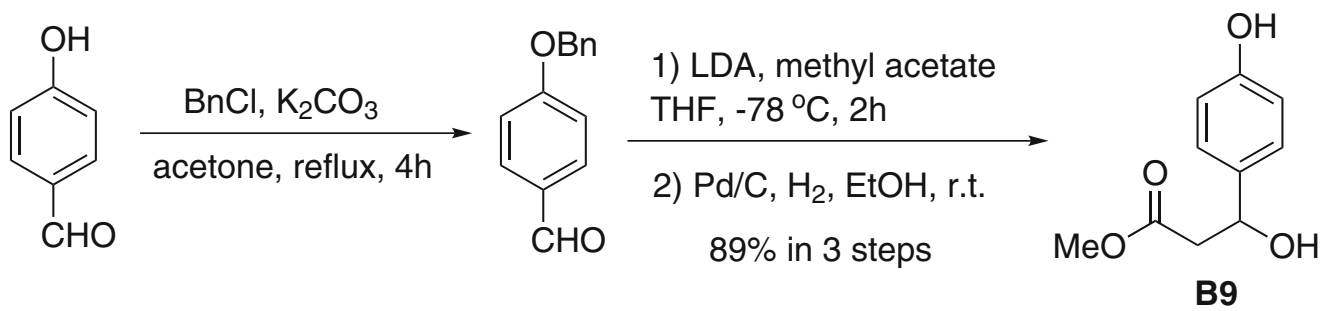<smiles>CCOC(=O)CC(O)c1ccc(OC)cc1</smiles>

Scheme 2 Synthetic route to compounds B9 and B10

refluxed for $4 \mathrm{~h}$. Cooled to room temperature, $20 \mathrm{~mL}$ icewater was added and extracted with EtOAc $(3 \times 100 \mathrm{~mL})$. The organic layers were combined and washed by brine $(3 \times 50 \mathrm{~mL})$, dried over $\mathrm{Na}_{2} \mathrm{SO}_{4}(\mathrm{~s})$, then evaporated the solvent under the reduced pressure to give 4-benzyloxy benzldehyde as a light yellow solid (21 g, $99 \%)$. This compound was used in next step without further purification.

To a solution of freshly distilled diisopropylamine $(14 \mathrm{~mL}, 0.12 \mathrm{~mol})$ in $100 \mathrm{~mL}$ dry $\mathrm{THF}$ at $-78{ }^{\circ} \mathrm{C}$ was added $n$-BuLi (50 mL of $2 \mathrm{M}$ in hexane, $0.1 \mathrm{~mol}$ ) and stirred for $15 \mathrm{~min}$. Freshly distilled methyl acetate (8.6 mL, $0.11 \mathrm{~mol}$ ) was added. The reaction stirred for $1 \mathrm{~h}$ at $-78{ }^{\circ} \mathrm{C}$, and 4-benzyloxyl benzldehyde (21 g, $\left.0.1 \mathrm{~mol}\right)$ in $100 \mathrm{~mL}$ dry THF was added. After $1 \mathrm{~h}$ at $-78^{\circ} \mathrm{C}$, the reaction was quenched with saturated $\mathrm{NH}_{4} \mathrm{Cl}$ aqueous solution $(50 \mathrm{~mL})$, warmed to room temperature, and stirred for an additional $6 \mathrm{~h}$. The solution was extracted with EtOAc $(3 \times 100 \mathrm{~mL})$, and the organic layers were combined and washed by brine $(3 \times 50 \mathrm{~mL})$, dried over $\mathrm{Na}_{2} \mathrm{SO}_{4}$ (s), evaporated the solvent under the reduced pressure to give crude product as a yellow solid, which was purified by flash chromatography with petroleum ether/ EtOAc (20/1) to give methyl 3-(4-(benzyloxy)phenyl)-3hydroxypropanoate $26 \mathrm{~g}(91 \%)$ as a white solid. ${ }^{1} \mathrm{H}$ NMR $\left(400 \mathrm{MHz}, \mathrm{CDCl}_{3}\right) \delta 7.29(\mathrm{~m}, 7 \mathrm{H}), 6.96(\mathrm{dd}, J=1.9$, $8.0 \mathrm{~Hz}, 2 \mathrm{H}), 5.08$ (dd, $J=3.7,9.2 \mathrm{~Hz}, 1 \mathrm{H}), 5.06(\mathrm{~s}, 2 \mathrm{H})$, $3.72(\mathrm{~s}, 3 \mathrm{H}), 2.66-2.80(\mathrm{~m}, 2 \mathrm{H})$.

To a solution of methyl 3-(4-(benzyloxy)phenyl)-3-hydroxypropanoate $(5 \mathrm{~g}, 17.48 \mathrm{mmol})$ in $50 \mathrm{~mL} \mathrm{EtOH}$, $500 \mathrm{mg} 10 \% \mathrm{Pd} / \mathrm{C}$ was added, which then stirred under $\mathrm{H}_{2}$ atmosphere overnight at room temperature. The reaction mixture was passed through a short pad of Celite to remove $\mathrm{Pd} / \mathrm{C}$ and evaporated the solvent under the reduced pressure to give $3.4 \mathrm{~g}$ (100\%) of $\mathbf{B 9}$ as a white foam. ${ }^{1} \mathrm{H} \mathrm{NMR}$ $\left(400 \mathrm{MHz}, \mathrm{CDCl}_{3}\right) \delta 7.22(\mathrm{~d}, J=8.5 \mathrm{~Hz}, 2 \mathrm{H}), 6.78(\mathrm{~d}$, $J=8.5 \mathrm{~Hz}, 2 \mathrm{H}), 5.07$ (dd, $J=3.7,9.2 \mathrm{~Hz}, 1 \mathrm{H}), 3.72$ (s, $3 \mathrm{H}), 2.66-2.81(\mathrm{~m}, 2 \mathrm{H})$. 
Table 2 In vitro anti-tumor assay of the synthetic compounds

\begin{tabular}{llllll}
\hline Entry & $\mathrm{IC}_{50}(\mu \mathrm{M})$ & & & & \\
\cline { 2 - 6 } & $\mathrm{HL}-60$ & SMMC-7721 & A-549 & MCF-7 & SW480 \\
\hline 5 & $>40$ & $>40$ & $>40$ & $>40$ & $>40$ \\
6 & $>40$ & $>40$ & $>40$ & $>40$ & $>40$ \\
7 & $>40$ & $>40$ & $>40$ & $>40$ & $>40$ \\
8 & $>40$ & $>40$ & $>40$ & $>40$ & $>40$ \\
9 & $>40$ & $>40$ & $>40$ & $>40$ & $>40$ \\
10 & $>40$ & $>40$ & $>40$ & $>40$ & $>40$ \\
11 & $>40$ & $>40$ & $>40$ & $>40$ & $>40$ \\
12 & $>40$ & $>40$ & $>40$ & $>40$ & $>40$ \\
13 & $>40$ & $>40$ & $>40$ & $>40$ & $>40$ \\
14 & $>40$ & $>40$ & $>40$ & $>40$ & $>40$ \\
15 & $>40$ & $>40$ & $>40$ & $>40$ & $>40$ \\
16 & $>40$ & $>40$ & $>40$ & $>40$ & $>40$ \\
DPP & 1.05 & 6.76 & 6.01 & 15.38 & 16.31 \\
Taxol & $<0.008$ & $<0.008$ & $<0.008$ & $<0.008$ & $<0.008$ \\
\hline
\end{tabular}

B10: To a solution of B13 (10 g, $44.6 \mathrm{mmol})$ in anhydrous $\mathrm{MeOH}, \mathrm{MeONa}(240 \mathrm{mg}, 4.46 \mathrm{mmol}$ ) was added. The mixture was stirred at $40{ }^{\circ} \mathrm{C}$ for $24 \mathrm{~h}$. Evaporated the solvent under the reduced pressure, the residue was dissolved in $100 \mathrm{~mL}$ EtOAc, washed by water $(3 \times 50 \mathrm{~mL})$, brine $(3 \times 50 \mathrm{~mL})$, dried over $\mathrm{Na}_{2} \mathrm{SO}_{4}(\mathrm{~s})$. Evaporated the solvent under the reduced pressure to give $9.28 \mathrm{~g}(100 \%)$ of $\mathbf{B 1 4}$ as a light yellow oil. ${ }^{1} \mathrm{H}$ NMR $\left(400 \mathrm{MHz}, \mathrm{CDCl}_{3}\right) \delta$ $7.91(\mathrm{~d}, J=8.8 \mathrm{~Hz}, 2 \mathrm{H}), 6.93(\mathrm{~d}, J=8.8 \mathrm{~Hz}, 2 \mathrm{H}), 3.95$ (s, $2 \mathrm{H}), 3.86(\mathrm{~s}, 3 \mathrm{H}), 3.73$ (s, 3H).

To a solution of $\mathbf{B 1 4}(5 \mathrm{~g}, 24 \mathrm{mmol})$ in $30 \mathrm{~mL} \mathrm{MeOH}$ at $0{ }^{\circ} \mathrm{C}$ was added $\mathrm{NaBH}_{4}(1.82 \mathrm{~g}, 48 \mathrm{mmol})$, the mixture was stirred at $0{ }^{\circ} \mathrm{C}$ for $2 \mathrm{~h}$. The reaction was quenched with saturated $\mathrm{NH}_{4} \mathrm{Cl}$ aqueous solution $(5 \mathrm{~mL})$ at $0{ }^{\circ} \mathrm{C}$. The resulting mixture was extracted with EtOAc $(3 \times 30 \mathrm{~mL})$, and the organic layers were combined and washed by brine $(3 \times 50 \mathrm{~mL})$, dried over $\mathrm{Na}_{2} \mathrm{SO}_{4}(\mathrm{~s})$. Evaporated the solvent under the reduced pressure to give crude product as a colorless oil, and the crude product was purified by flash chromatography with petroleum ether/EtOAc (20/1) to give $4.7 \mathrm{~g}(93 \%)$ of $\mathbf{B 1 0}$ as a colorless oil. ${ }^{1} \mathrm{H}$ NMR $\left(400 \mathrm{MHz}, \mathrm{CDCl}_{3}\right) \delta 7.30(\mathrm{dd}, J=2.8,8.6 \mathrm{~Hz}, 2 \mathrm{H}), 6.90$ $(\mathrm{dd}, J=2.8,8.6 \mathrm{~Hz}, 2 \mathrm{H}), 5.08(\mathrm{dd}, J=3.7,9.3 \mathrm{~Hz}, 1 \mathrm{H})$, $3.80(\mathrm{~s}, 3 \mathrm{H}), 3.71(\mathrm{~s}, 3 \mathrm{H}), 2.65-2.80(\mathrm{~m}, 2 \mathrm{H})$.

\subsection{General Procedure for the Preparation of L-AA Lactone Derivatives (5-16)}

Method A [26] for lactones 5, 7, 13, 14, 15: To L-AA (3 eq.) in $2 \mathrm{~mL}$ water was added corresponding alcohol B1 or $\mathbf{B 3}$ or $\mathbf{B 9}$ or $\mathbf{B 1 0}(0.5 \mathrm{mmol}, 1$ eq.), and the solution stirred at $50{ }^{\circ} \mathrm{C}$ for $72 \mathrm{~h}$. The reaction was evaporated under reduced pressure and the residue was purified by column chromatography with DCM/MeOH (50/1-20/1) to afford lactone 5 or 7 or $\mathbf{1 3}, \mathbf{1 5}$ or 14, respectively.

$5(85 \%)$, white foam: ${ }^{1} \mathrm{H}$ NMR $\left(600 \mathrm{MHz}\right.$, acetone- $\left.d_{6}\right)$ $\delta 8.31(\mathrm{~s}, 1 \mathrm{H}), 7.14(\mathrm{~d}, J=8.5 \mathrm{~Hz}, 2 \mathrm{H}), 6.73(\mathrm{~d}$, $J=8.5 \mathrm{~Hz}, 2 \mathrm{H}), 5.86(\mathrm{~s}, 1 \mathrm{H}), 4.66(\mathrm{~s}, 1 \mathrm{H}), 4.45(\mathrm{~s}, 1 \mathrm{H})$, $4.30(\mathrm{~s}, 1 \mathrm{H}), 4.09(\mathrm{dd}, J=9.7,5.5 \mathrm{~Hz}, 1 \mathrm{H}), 4.00(\mathrm{dd}$, $J=9.7,3.1 \mathrm{~Hz}, 1 \mathrm{H}), 3.77(\mathrm{~s}, 1 \mathrm{H}), 3.09(\mathrm{~d}, J=13.5 \mathrm{~Hz}$, $1 \mathrm{H}), 2.92(\mathrm{~d}, J=13.5 \mathrm{~Hz}, 1 \mathrm{H}) .{ }^{13} \mathrm{C}$ NMR $(150 \mathrm{MHz}$, acetone- $\left.d_{6}\right) \delta 175.88,157.41,132.83,125.75,115.60$, $108.31,86.97,80.77,75.51,75.45,55.05$, 40.62. HRESIMS $\mathrm{m} / \mathrm{z}, 305.0636$ (calcd for $\mathrm{C}_{13} \mathrm{H}_{14} \mathrm{O}_{7}[\mathrm{M}+\mathrm{Na}]^{+}$, 305.0632).

$7(35 \%)$, yellow oil: ${ }^{1} \mathrm{H}$ NMR (600 MHz, acetone- $\left.d_{6}\right) \delta$ $6.81(\mathrm{~d}, J=2.0 \mathrm{~Hz}, 1 \mathrm{H}), 6.70(\mathrm{~d}, J=8.1 \mathrm{~Hz}, 1 \mathrm{H}), 6.65$ (dd, $J=8.1,2.0 \mathrm{~Hz}, 1 \mathrm{H}), 5.85(\mathrm{~s}, 1 \mathrm{H}), 4.66(\mathrm{~s}, 1 \mathrm{H}), 4.42$ (s, 1H), $4.30(\mathrm{~s}, 1 \mathrm{H}), 4.09(\mathrm{dd}, J=9.7,5.5 \mathrm{~Hz}, 1 \mathrm{H}), 3.99$ $(\mathrm{dd}, J=9.7,3.2 \mathrm{~Hz}, 1 \mathrm{H}), 3.04(\mathrm{~d}, J=13.4 \mathrm{~Hz}, 1 \mathrm{H}), 2.87$ $(\mathrm{s}, 1 \mathrm{H}), 2.85(\mathrm{~d}, J=13.4 \mathrm{~Hz}, 1 \mathrm{H}) .{ }^{13} \mathrm{C}$ NMR $(150 \mathrm{MHz}$, acetone- $\left.d_{6}\right) \delta 175.95,145.25,145.17,126.45,123.30$, 118.81, 115.60, 108.34, 86.97, 80.68, 75.49, 75.47, 40.90. HRESIMS $m / z 333.0377$ (calcd for $\mathrm{C}_{13} \mathrm{H}_{14} \mathrm{O}_{8}[\mathrm{M}+\mathrm{Na}]^{+}$, 333.0383).

13 (35\%), white foam: ${ }^{1} \mathrm{H}$ NMR $(600 \mathrm{MHz}$, acetone$\left.d_{6}\right) \delta 8.67(\mathrm{~s}, 1 \mathrm{H}), 7.29(\mathrm{~d}, J=8.7 \mathrm{~Hz}, 2 \mathrm{H}), 6.86(\mathrm{~d}$, $J=8.7 \mathrm{~Hz}, 2 \mathrm{H}), 6.56(\mathrm{~s}, 1 \mathrm{H}), 4.79(\mathrm{~s}, 1 \mathrm{H}), 4.25-4.40(\mathrm{~m}$, $2 \mathrm{H}), 4.21(\mathrm{dd}, J=9.7,6.0 \mathrm{~Hz}, 1 \mathrm{H}), 4.02(\mathrm{dd}, J=9.7$, $3.7 \mathrm{~Hz}, 1 \mathrm{H}), 3.90(\mathrm{~s}, 1 \mathrm{H}), 3.17(\mathrm{dd}, J=17.3,13.2 \mathrm{~Hz}$, $1 \mathrm{H}), 2.90$ (dd, $J=17.3,8.5 \mathrm{~Hz}, 1 \mathrm{H}) .{ }^{13} \mathrm{C} \mathrm{NMR}(150 \mathrm{MHz}$, acetone- $\left.d_{6}\right) \delta 174.37,172.06,158.70,130.91,124.09$, 116.48, 106.38, 90.12, 88.98, 76.19, 74.81, 45.66, 33.97. HRESIMS $\mathrm{m} / \mathrm{z} 321.0607$ (calcd for $\mathrm{C}_{15} \mathrm{H}_{14} \mathrm{O}_{8}[\mathrm{M}-\mathrm{H}]^{-}$, 321.0616).

15 (40\%), light yellow oil: ${ }^{1} \mathrm{H}$ NMR (600 MHz, acetone- $\left.d_{6}\right) \delta 8.46(\mathrm{~s}, 1 \mathrm{H}), 7.25(\mathrm{~d}, J=9.0 \mathrm{~Hz}, 2 \mathrm{H}), 6.74(\mathrm{~d}$, $J=9.0 \mathrm{~Hz}, 2 \mathrm{H}), 5.78(\mathrm{~s}, 1 \mathrm{H}), 4.72(\mathrm{~s}, 1 \mathrm{H}), 4.26(\mathrm{~m}, 1 \mathrm{H})$, $4.02(\mathrm{~m}, 2 \mathrm{H}), 3.62(\mathrm{~m}, 1 \mathrm{H}), 3.47(\mathrm{~s}, 3 \mathrm{H}), 3.29(\mathrm{~d}$, $J=1.5 \mathrm{~Hz}, 2 \mathrm{H}$ ). HRESIMS $\mathrm{m} / \mathrm{z} 377.0845$ (calcd for $\left.\mathrm{C}_{16} \mathrm{H}_{18} \mathrm{O}_{9}[\mathrm{M}+\mathrm{Na}]^{+}, 377.0843\right)$.

$14(30 \%)$, white foam: ${ }^{1} \mathrm{H}$ NMR $(600 \mathrm{MHz}$, acetone$\left.d_{6}\right) \delta 8.67(\mathrm{~s}, 1 \mathrm{H}), 7.38(\mathrm{~d}, J=9 \mathrm{~Hz}, 2 \mathrm{H}), 6.96(\mathrm{~d}$, $J=9 \mathrm{~Hz}, 2 \mathrm{H}), 6.56(\mathrm{~s}, 1 \mathrm{H}), 4.79(\mathrm{~s}, 1 \mathrm{H}), 4.25-4.40(\mathrm{~m}$, $2 \mathrm{H}), 4.21(\mathrm{dd}, J=9.7,6.0 \mathrm{~Hz}, 1 \mathrm{H}), 4.02(\mathrm{dd}, J=9.7$, $3.7 \mathrm{~Hz}, 1 \mathrm{H}), 3.90(\mathrm{~s}, 1 \mathrm{H}), 3.81$ (s, $3 \mathrm{H}), 3.17$ (dd, $J=17.3$, $13.2 \mathrm{~Hz}, 1 \mathrm{H}), 2.90(\mathrm{dd}, J=17.3,8.5 \mathrm{~Hz}, 2 \mathrm{H}) .{ }^{13} \mathrm{C} \mathrm{NMR}$ $\left(150 \mathrm{MHz}\right.$, acetone- $\left.d_{6}\right) \delta 174.32,171.98,160.88,130.87$, 125.34, 115.02, 106.38, 90.05, 88.95, 76.22, 74.78, 55.62, 45.57, 33.98. HRESIMS $m / z 359.0740$ (calcd for $\mathrm{C}_{16} \mathrm{H}_{16} \mathrm{O}_{8}$ $\left.[\mathrm{M}+\mathrm{Na}]^{+}, 359.0737\right)$.

Method B for lactones $6,8,9,10,11,12$ : To L-AA (4 eq.) in $4 \mathrm{~mL}$ phosphate-citrate buffer $(p \mathrm{H}=5.0)$ was added corresponding alcohol $\mathbf{B 2}$ or $\mathbf{B 4}$ or $\mathbf{B 5}$ or $\mathbf{B 6}$ or $\mathbf{B 7}$ or $\mathbf{B 8}$ (1 mmol, 1 eq.), and the solution stirred at $40-60{ }^{\circ} \mathrm{C}$ 
for $36-72 \mathrm{~h}$. The reaction was evaporated under reduced pressure and the residue was purified by colum chromatography with DCM/MeOH (50/1-20/1) to afford lactone 6 or $\mathbf{8}$ or 9 or $\mathbf{1 0}$ or $\mathbf{1 1}$ or $\mathbf{1 2}$, respectively.

$6(53 \%)$, yellow foam: ${ }^{1} \mathrm{H}$ NMR $(600 \mathrm{MHz}$, acetone$\left.d_{6}\right) \delta 7.22(\mathrm{~d}, J=8.7 \mathrm{~Hz}, 2 \mathrm{H}), 6.82(\mathrm{~d}, J=8.7 \mathrm{~Hz}, 2 \mathrm{H})$, $5.90(\mathrm{~s}, 1 \mathrm{H}), 4.68(\mathrm{~s}, 1 \mathrm{H}), 4.50(\mathrm{~s}, 1 \mathrm{H}), 4.31(\mathrm{dd}, J=5.3$, $3.2 \mathrm{~Hz}, 1 \mathrm{H}$ ), 4.09 (dd, $J=9.7,5.5 \mathrm{~Hz}, 1 \mathrm{H}), 4.01$ (dd, $J=9.7,3.1 \mathrm{~Hz}, 1 \mathrm{H}), 3.82(\mathrm{~s}, 1 \mathrm{H}), 3.77(\mathrm{~s}, 3 \mathrm{H}), 3.11(\mathrm{~d}$, $J=13.6 \mathrm{~Hz}, 1 \mathrm{H}), 2.96(\mathrm{~d}, J=13.6 \mathrm{~Hz}, 1 \mathrm{H}) .{ }^{13} \mathrm{C} \mathrm{NMR}$ $\left(150 \mathrm{MHz}\right.$, acetone- $\left.d_{6}\right) \delta 175.74,159.76,132.78,127.07$, 114.09, 108.30, 87.03, 80.64, 75.57, 75.44, 55.42, 40.51. HRESIMS $m / z \quad 319.0788$ (calcd for $\mathrm{C}_{14} \mathrm{H}_{16} \mathrm{O}_{7}[\mathrm{M}+\mathrm{Na}]^{+}$, 319.0787).

$8(72 \%)$, white foam: ${ }^{1} \mathrm{H}$ NMR $\left(600 \mathrm{MHz}\right.$, acetone- $\left.d_{6}\right)$ $\delta 6.83(\mathrm{~d}, J=1.4 \mathrm{~Hz}, 1 \mathrm{H}), 6.76(\mathrm{dt}, J=16.3,4.7 \mathrm{~Hz}$, $2 \mathrm{H}), 5.96(\mathrm{~d}, J=1.7 \mathrm{~Hz}, 2 \mathrm{H}), 5.90(\mathrm{~s}, 1 \mathrm{H}), 4.71(\mathrm{~s}, 1 \mathrm{H})$, $4.56(\mathrm{~s}, 1 \mathrm{H}), 4.34(\mathrm{dd}, J=4.6,3.2 \mathrm{~Hz}, 1 \mathrm{H}), 4.11$ (dd, $J=9.7,5.5 \mathrm{~Hz}, 1 \mathrm{H}), 4.03$ (m, overlaped, $2 \mathrm{H}), 3.08$ (d, $J=13.7 \mathrm{~Hz}, 1 \mathrm{H}), 2.96(\mathrm{~d}, J=13.7 \mathrm{~Hz}, 1 \mathrm{H}) .{ }^{13} \mathrm{C} \mathrm{NMR}$ $\left(150 \mathrm{MHz}\right.$, acetone- $\left.d_{6}\right) \delta 175.49,148.05,147.55,129.05$, $124.87,111.96,108.47,108.31,101.88,87.10,80.48$, 75.70, 75.41, 40.91. HRESIMS $m / z, 333.0586$ (calcd for $\left.\mathrm{C}_{14} \mathrm{H}_{14} \mathrm{O}_{8}[\mathrm{M}+\mathrm{Na}]^{+}, 333.0581\right)$.

$9(65 \%)$, colorless oil: main isomer: ${ }^{1} \mathrm{H}$ NMR $\left(600 \mathrm{MHz}\right.$, acetone- $\left.d_{6}\right) \delta 8.35(\mathrm{~s}, 1 \mathrm{H}), 7.17(\mathrm{~d}, J=8.8 \mathrm{~Hz}$, $2 \mathrm{H}), 6.74(\mathrm{~d}, J=8.8 \mathrm{~Hz}, 2 \mathrm{H}), 5.71(\mathrm{~s}, 1 \mathrm{H}), 4.57$ (d, $J=3.7 \mathrm{~Hz}, 1 \mathrm{H}), 4.38(\mathrm{~s}, 1 \mathrm{H}), 4.23(\mathrm{~d}, J=2.3 \mathrm{~Hz}, 1 \mathrm{H})$, $4.04(\mathrm{dd}, J=9.7,5.4 \mathrm{~Hz}, 1 \mathrm{H}), 3.95(\mathrm{dd}, J=9.7,2.9 \mathrm{~Hz}$, $1 \mathrm{H}), 3.34(\mathrm{q}, J=7.2 \mathrm{~Hz}, 1 \mathrm{H}), 3.13(\mathrm{~s}, 1 \mathrm{H}), 1.40(\mathrm{~d}$, $J=7.2 \mathrm{~Hz}, 3 \mathrm{H}) .{ }^{13} \mathrm{C} \mathrm{NMR}\left(150 \mathrm{MHz}\right.$, acetone- $\left.d_{6}\right) \delta$ $175.36,157.37,131.85,129.12,116.30,115.48,108.61$, 86.28, 81.86, 75.30, 75.25, 43.24, 16.40. HRESIMS $\mathrm{m} / \mathrm{z}$ 295.0816 (calcd for $\mathrm{C}_{14} \mathrm{H}_{16} \mathrm{O}_{7}[\mathrm{M}-\mathrm{H}]^{-}, 295.0823$ ).

$10(42 \%)$, colorless oil, main isomer: ${ }^{1} \mathrm{H}$ NMR $\left(600 \mathrm{MHz}\right.$, acetone- $\left.d_{6}\right) \delta 7.29(\mathrm{~d}, J=8.8 \mathrm{~Hz}, 2 \mathrm{H}), 6.82(\mathrm{~d}$, $J=8.8 \mathrm{~Hz}, 2 \mathrm{H}), 5.69$ (s, 1H), 4.70 (s, 1H), 4.39 (d, $J=6.1 \mathrm{~Hz}, 1 \mathrm{H}), 4.32(\mathrm{dd}, J=5.0,3.1 \mathrm{~Hz}, 1 \mathrm{H}), 4.05-4.08$ (m, overlaped, 1H), 3.97 (dd, $J=11.5,3.0 \mathrm{~Hz}, 1 \mathrm{H}), 3.83$ (s, 1H), 3.77 (s, 3H), 3.23 (q, $J=7.3 \mathrm{~Hz}, 1 \mathrm{H}), 1.51$ (d, $J=7.3 \mathrm{~Hz}, 3 \mathrm{H}) .{ }^{13} \mathrm{C} \mathrm{NMR}\left(150 \mathrm{MHz}\right.$, acetone- $\left.d_{6}\right) \delta$ $176.34,159.64,131.76,130.42,114.69,113.74,109.15$, 87.03, 86.29, 75.31, 75.10, 55.41, 43.25, 16.39. HRESIMS $m / z 333.0949$ (calcd for $\mathrm{C}_{15} \mathrm{H}_{18} \mathrm{O}_{7}[\mathrm{M}+\mathrm{Na}]^{+}, 333.0945$ ).

$11(78 \%)$, colorless oil: ${ }^{1} \mathrm{H}$ NMR (600 MHz, acetone$\left.d_{6}\right) \delta 7.57(\mathrm{~s}, 1 \mathrm{H}), 6.92(\mathrm{~d}, J=1.9 \mathrm{~Hz}, 1 \mathrm{H}), 6.80-6.67(\mathrm{~m}$, 2H), $5.87(\mathrm{~s}, 1 \mathrm{H}), 4.69(\mathrm{~s}, 1 \mathrm{H}), 4.51(\mathrm{~s}, 1 \mathrm{H}), 4.31(\mathrm{~m}, 1 \mathrm{H})$, $4.09(\mathrm{dd}, J=9.8,5.4 \mathrm{~Hz}, 1 \mathrm{H}), 4.01(\mathrm{dd}, J=9.7,3.0 \mathrm{~Hz}$, $1 \mathrm{H}), 3.82(\mathrm{~s}, 1 \mathrm{H}), 3.78(\mathrm{~s}, 3 \mathrm{H}), 3.11(\mathrm{~d}, J=13.5 \mathrm{~Hz}, 1 \mathrm{H})$, $2.94(\mathrm{~d}, J=13.5 \mathrm{~Hz}, 1 \mathrm{H}) .{ }^{13} \mathrm{C}$ NMR $(150 \mathrm{MHz}$, acetone$\left.d_{6}\right) \delta 175.94,147.61,146.55,126.16,124.37,115.33$, $115.21,108.30,86.97,80.79,75.48,75.34,56.14,41.03$.
HRESIMS $m / z \quad 335.0742$ (calcd for $\mathrm{C}_{14} \mathrm{H}_{16} \mathrm{O}_{8}$ $\left.[\mathrm{M}+\mathrm{Na}]^{+}, 335.0737\right)$.

$12(58 \%)$, white foam: ${ }^{1} \mathrm{H}$ NMR $(600 \mathrm{MHz}$, acetone$\left.d_{6}\right) \delta 8.22(\mathrm{~s}, 1 \mathrm{H}), 7.05(\mathrm{~d}, J=1.9 \mathrm{~Hz}, 1 \mathrm{H}), 6.92(\mathrm{~d}$, $J=1.8 \mathrm{~Hz}, 1 \mathrm{H}), 5.93(\mathrm{~s}, 1 \mathrm{H}), 4.73(\mathrm{~s}, 1 \mathrm{H}), 4.60(\mathrm{~s}, 1 \mathrm{H})$, $4.35(\mathrm{dd}, J=5.2,3.3 \mathrm{~Hz}, 1 \mathrm{H}), 4.11(\mathrm{dd}, J=9.8,5.5 \mathrm{~Hz}$, $1 \mathrm{H}), 4.03(\mathrm{dd}, J=9.8,3.0 \mathrm{~Hz}, 2 \mathrm{H}), 3.81(\mathrm{~s}, 3 \mathrm{H}), 3.07(\mathrm{~d}$, $J=13.8 \mathrm{~Hz}, 1 \mathrm{H}), 2.97$ (m, overlaped, $1 \mathrm{H}) .{ }^{13} \mathrm{C} \mathrm{NMR}$ $\left(150 \mathrm{MHz}\right.$, acetone- $\left.d_{6}\right) \delta 175.50,148.28,143.97,127.58$, $127.50,114.28,108.59,108.29,87.26,80.38,75.67$, 75.38, 56.61, 40.46. HRESIMS $\mathrm{m} / z$ 388.9870, 390.9851 $(\mathrm{M}+2-\mathrm{H})\left(\right.$ calcd for $\left.\mathrm{C}_{14} \mathrm{H}_{15} \mathrm{BrO}_{8}[\mathrm{M}-\mathrm{H}]^{-}, 388.9878\right)$.

Method C [27] for lactone 16: To L-AA (2.3 eq.) in $10 \mathrm{~mL}$ phosphate-citrate buffer ( $\mathrm{PH} \mathrm{5.0)}$ was added corresponding alcohol $\mathbf{B 1 1}$ ( $1 \mathrm{mmol}, 1$ eq.) in $1 \mathrm{~mL} \mathrm{EtOH}$, and the solution stirred at room temperature for $8 \mathrm{~h}$. The reaction mixture was extracted with EtOAc, the extract was washed with water, dried over $\mathrm{Na}_{2} \mathrm{SO}_{4}(\mathrm{~s})$, and the solvent was evaporated under reduced pressure and the residue was purified by column chromatography with DCM/MeOH (10/1) to afford lactone $16(57 \%)$ a gray foam. ${ }^{1} \mathrm{H}$ NMR $\left(600 \mathrm{MHz}\right.$, acetone- $\left.d_{6}\right) \quad \delta \quad 10.18 \quad(\mathrm{~s}, 1 \mathrm{H}), 7.66 \quad(\mathrm{~d}$, $J=8.0 \mathrm{~Hz}, 1 \mathrm{H}), 7.37(\mathrm{~d}, J=8.0 \mathrm{~Hz}, 1 \mathrm{H}), 7.29$ (d, $J=2.3 \mathrm{~Hz}, 1 \mathrm{H}), 7.07(\mathrm{t}, J=8.0,1.0 \mathrm{~Hz}, 1 \mathrm{H}), 6.98(\mathrm{t}$, $J=8.0,1.0 \mathrm{~Hz}, 1 \mathrm{H}), 5.92(\mathrm{~s}, 1 \mathrm{H}), 4.65(\mathrm{~s}, 1 \mathrm{H}), 4.50(\mathrm{~s}$, $1 \mathrm{H}), 4.28(\mathrm{~m}, 1 \mathrm{H}), 4.10(\mathrm{dd}, J=9.7,5.5 \mathrm{~Hz}, 1 \mathrm{H}), 4.01$ (dd, $J=9.7, \quad 3.2 \mathrm{~Hz}, 1 \mathrm{H}), 3.91$ (s, 1H), 3.39 (d, $J=14.3 \mathrm{~Hz}, 1 \mathrm{H}), 3.25$ (d, $J=14.3 \mathrm{~Hz}, 1 \mathrm{H}) .{ }^{13} \mathrm{C} \mathrm{NMR}$ $\left(150 \mathrm{MHz}\right.$, acetone- $\left.d_{6}\right) \delta 176.47,137.13,129.06,126.46$, $122.00,120.06,119.52,111.99,108.53,107.89,87.24$, 80.22, 75.47, 75.45, 31.22. HRESIMS m/z 328.0795 (calcd for $\left.\mathrm{C}_{15} \mathrm{H}_{15} \mathrm{NO}_{6}[\mathrm{M}+\mathrm{Na}]^{+}, 328.0792\right)$.

Acknowledgments This work was financially supported by the National Natural Science Foundation of China (No. U0932602) and the National Basic Research Program of China (973 Program No. 2011CB915503). All authors do not have any financial/commercial conflicts of interest.

Conflict of interest The authors declare no conflict of interest.

Open Access This article is distributed under the terms of the Creative Commons Attribution License which permits any use, distribution, and reproduction in any medium, provided the original author(s) and the source are credited.

\section{References}

1. M.W. Davey, M.V. Montagu, D. Inze, M. Sanmartin, A. Kanellis, N. Smirnoff, I.J. Benzie, J.J. Strain, D. Favell, J. Fletcher, J. Sci. Food Agric. 80, 825-860 (2000)

2. T. Gazivoda, M. Plevnik, J. Plavec, S. Kraljević, M. Kralj, K. Pavelić, J. Balzarini, E.D. Clercq, M. Mintas, S. Raić-Malić, Bioorg. Med. Chem. 13, 131-139 (2005) 
3. T. Gazivoda, S. Raić-Malić, M. Marjanović, M. Kralj, K. Pavelić, J. Balzarini, E.D. Clercq, M. Mintas, Bioorg. Med. Chem. 15, 749-758 (2007)

4. T. Gazivoda, M. Šokčević, M. Kralj, L. Šuman, K. Pavelić, E. De Clercq, G. Andrei, R. Snoeck, J. Balzarini, M. Mintas, S. RaićMalić, J. Med. Chem. 50, 4105-4112 (2007)

5. S. Raić-Malić, A. Hergold-Brundić, A. Nagl, M. Grdiša, K. Pavelić, E. De Clercq, M. Mintas, J. Med. Chem. 42, 2673-2678 (1999)

6. M.W. Roomi, D. House, M. Eckert-Maksić, Z.B. Maksić, C.S. Tsao, Cancer Lett. 122, 93-99 (1998)

7. K. Morisaki, S. Ozaki, Chem. Pharm. Bull. 44, 1647-1655 (1996)

8. I. Yamamoto, A. Tai, Y. Fujinami, K. Sasaki, S. Okazaki, J. Med. Chem. 45, 462-468 (2001)

9. A. Tai, S. Goto, Y. Ishiguro, K. Suzuki, T. Nitoda, I. Yamamoto, Bioorg. Med. Chem. Lett. 14, 623-627 (2004)

10. S. Manfredini, B. Pavan, S. Vertuani, M. Scaglianti, D. Compagnone, C. Biondi, A. Scatturin, S. Tanganelli, L. Ferraro, P. Prasad, A. Dalpiaz, J. Med. Chem. 45, 559-562 (2001)

11. S. Palma, R.H. Manzo, D. Allemandi, L. Frationi, P.L. Nostro, J. Pharm. Sci. 91, 1810-1816 (2002)

12. M.H. Sung, C. Park, S.C. Kim, G.S. Park, H. Uyama, H.R. Poo, J.J. Song. WO2006001567A1, (2006)

13. D.J. Ko, J.W. Choi, B.S. Seo, M.J. Lim. KR2012017299A, (2012)

14. N.G. Kesinger, J.F. Stevens, Phytochemistry 70, 1930-1939 (2009)

15. Z. Zhang, X. Liu, X. Zhang, J. Liu, Y. Hao, X. Yang, Y. Wang, Arch. Pharm. Res. 34, 801-810 (2011)
16. A. Tai, Bitamin 87, 70-80 (2013)

17. J.C. Yvin, A.M. Chevolot-Magueur, L. Chevolot, J.Y. Lallemand, P. Potier, J. Guilhem, J. Am. Chem. Soc. 104, 4497-4498 (1982)

18. T. Iwagawa, T. Hase, Phytochemistry 23, 2299-2301 (1984)

19. J. He, X.Q. Chen, M.M. Li, Y. Zhao, G. Xu, X. Cheng, L.Y. Peng, M.J. Xie, Y.T. Zheng, Y.P. Wang, Org. Lett. 11, 1397-1400 (2009)

20. L.B. Dong, J. Yang, J. He, H.R. Luo, X.D. Wu, X. Deng, L.Y. Peng, X. Cheng, Q.S. Zhao, Chem. Comm. 48, 9038-9040 (2012)

21. J.T. Cheng, F. Liu, X.N. Li, X.D. Wu, L.B. Dong, L.Y. Peng, S.X. Huang, J. He, Q.S. Zhao, Org. Lett. 15, 2438-2441 (2013)

22. F. Liu, X.D. Wu, J. He, X. Deng, L.Y. Peng, H.R. Luo, Q.S. Zhao, Tetrahedron Lett. 54, 4555-4557 (2013)

23. X.Y. Zhang, L.B. Dong, F. Liu, X.D. Wu, J. He, L.Y. Peng, H.R. Luo, Q.S. Zhao, Nat. Prod. Bioprospec. 3, 52-55 (2013)

24. X.L. Li, X. Cheng, L.M. Yang, R.R. Wang, Y.T. Zheng, W.L. Xiao, Y. Zhao, G. Xu, Y. Lu, Y. Chang, Q.T. Zheng, Q.S. Zhao, H.D. Sun, Org. Lett. 8, 1937-1940 (2006)

25. Z. Wang, K. Zhao, J. Fu, J. Zhang, W. Yin, Y. Tang, Org. Biomol. Chem. 11, 2093-2097 (2013)

26. A.J. Poss, R.K. Belter, J. Org. Chem. 53, 1535-1540 (1988)

27. A.M. Korolev, É.I. Lazhko, M.N. Preobrazhenskaya, Y. Bal'zarini, É. Klerk, Pharm. Chem. J. 25, 805-808 (1991) 